\title{
NONLINEAR HAMMERSTEIN EQUATIONS AND FUNCTIONS OF BOUNDED RIESZ-MEDVEDEV VARIATION
}

\author{
Jürgen Appell — Tomás Domínguez BenAVides
}

\begin{abstract}
In this paper we study the solvability of a nonlinear Hammerstein type integral equation in the space of functions of bounded RieszMedvedev variation. To this end, we derive a compactness criterion and apply Schauder's fixed point theorem to a suitable operator whose fixed points coincide with the solutions of the integral equation.
\end{abstract}

\section{Statement of the problem}

Consider the nonlinear integral equation of Hammerstein type

$$
f(s)=\int_{a}^{b} k(s, t) h(t, f(t)) d t+b(s) .
$$

We are interested in conditions on the given functions $k, h$, and $b$ under which the above equation admits a solution $f$ in some space of functions of generalized bounded variation. To this end, we reformulate (1.1) as usual as a fixed point problem $f=A f$ for the operator

$$
A f:=K H f+b,
$$

2010 Mathematics Subject Classification. 26A45, 26A46, 45G10, 47H10, 47H30.

Key words and phrases. Bounded variation; nonlinear integral equation; fixed point theorem; Orlicz space.

The second author is partially supported by MCIN, Grant MTM 2012-34847-C02-01, Andalusian Regional Government Grant FQM-127 and DAAD grant ID 57051545.

This research was done while the second author was visiting the University of Würzburg; hospitality of the Department of Mathematics is gratefully acknowledged. 
where

$$
H f(t)=h(t, f(t))
$$

is the (nonlinear) composition operator generated by the function $h:[a, b] \times \mathbb{R} \rightarrow \mathbb{R}$, and

$$
K g(s)=\int_{a}^{b} k(s, t) g(t) d t
$$

is the (linear) integral operator generated by the kernel $k:[a, b] \times[a, b] \rightarrow \mathbb{R}$. Of course, this idea is very old, and there is a wealth of existence theorems in the literature, obtained in this way by considering the operators (1.3) and (1.4) in the space $C([a, b])$ of continuous functions, or in spaces of measurable functions like the Lebesgue space $L_{p}([a, b])$ and the Orlicz space $L_{\phi}([a, b])$. On the other hand, to the best of our knowledge there are only very few papers on the solvability of (1.1) in spaces of functions of (classical or generalized) bounded variation, although these spaces frequently occur in applications. As a sample result, we mention [18], where the author considers BV-solutions of a nonlinear convolutiontype Volterra integral equation on the real line. More recently, Bugajewska, Bugajewski et al. [6]-[12] study equation (1.1) more systematically from the viewpoint of variation and prove existence of solutions in the spaces $\operatorname{BV}([a, b])$ (bounded Jordan variation, see $[15]), \mathrm{WBV}_{p}([a, b])$ (bounded Wiener variation, see [31]), or even $\Lambda \mathrm{BV}([a, b])$ (bounded Waterman variation, [27]-[30]).

The purpose of this paper is to prove existence of solutions of (1.1) in the spaces $\mathrm{BV}_{p}([a, b])$ of functions of bounded Riesz $p$-variation or, more generally, $\mathrm{BV}_{\phi}([a, b])$ of functions of bounded Medvedev $\phi$-variation. First we recall the definition and some properties of these spaces and derive a natural compactness criterion. Afterwards we impose some conditions on the given data $h$ and $k$ under which the operator (1.2) is continuous and compact, and leaves a closed ball in the space $\mathrm{BV}_{p}([a, b])$ or $\mathrm{BV}_{\phi}([a, b])$ invariant. By means of Schauder's fixed point theorem we obtain then existence of solutions of (1.1). Finally, we briefly sketch how our method could be extended to a larger class of equations if we define a measure of noncompactness in $\mathrm{BV}_{p}([a, b])$ and replace Schauder's theorem by Darbo's fixed point theorem [13].

\section{The Riesz-Medvedev variation}

We begin this section recalling the definition of the Riesz variation [24], [25] which contains the Jordan variation as special case. Throughout this paper, we take $[a, b]=[0,1]$ for simplicity of notation. 
Definition 2.1. Given a real number $p \geq 1$, the total Riesz variation of a function $f:[0,1] \rightarrow \mathbb{R}$ is defined to be the (possibly infinite) number

$$
\operatorname{Var}_{p}(f):=\sup \left\{\sum_{j=1}^{m} \frac{\left|f\left(t_{j}\right)-f\left(t_{j-1}\right)\right|^{p}}{\left(t_{j}-t_{j-1}\right)^{p-1}}: P=\left\{t_{0}, \ldots, t_{m}\right\}\right\},
$$

where the supremum is taken over all partitions $P=\left\{t_{0}, \ldots, t_{m}\right\}$ of the interval $[0,1]$. In case $\operatorname{Var}_{p}(f)<\infty$ we say that $f$ has bounded Riesz variation on $[0,1]$ and write $f \in \mathrm{BV}_{p}([0,1])$.

In what follows we write $\mathrm{BV}_{p}$ instead of $\mathrm{BV}_{p}([0,1])$, and similarly for other function spaces. It is not hard to show that $\mathrm{BV}_{p}$, equipped with the norm

$$
\|f\|_{\mathrm{BV}_{p}}:=|f(0)|+\operatorname{Var}_{p}(f)^{1 / p} \quad\left(f \in \mathrm{BV}_{p}\right),
$$

is a Banach space which is for $p>1$ continuously imbedded into the space $C$ of continuous functions with the usual maximum norm, see Corollary 2.3 below. Moreover, the chain of (strict) inclusions

$$
\text { Lip } \subset \mathrm{BV}_{p} \subset \mathrm{BV}_{q} \subset \mathrm{AC} \subset \mathrm{BV}
$$

holds for $1<q<p<\infty$, where Lip denotes the space of Lipschitz continuous functions, $\mathrm{AC}$ the space of absolutely continuous functions, and $\mathrm{BV}=\mathrm{BV}_{1}$ the classical space of functions of bounded Jordan variation [15].

The most interesting and useful property of the space $\mathrm{BV}_{p}$ is that it is isometrically isomorphic to the space $L_{p} \oplus_{1} \mathbb{R}$ in the following sense.

TheOrem 2.2. Let $1<p<\infty$. Then, a function $f$ belongs to $\mathrm{BV}_{p}$ if and only if $f$ is absolutely continuous and $f^{\prime} \in L_{p}$. Moreover,

$$
\operatorname{Var}_{p}(f)=\int_{0}^{1}\left|f^{\prime}(x)\right|^{p} d x
$$

and so

$$
\|f\|_{\mathrm{BV}_{p}}=|f(0)|+\left\|f^{\prime}\right\|_{L_{p}} .
$$

Theorem 2.2 is due to F. Riesz [25]. An analogous result for $p=1$ is of course not true, since functions from $\mathrm{BV}_{1}=\mathrm{BV}$ are usually not continuous, let alone absolutely continuous. However, the decomposition $\mathrm{AC}=L_{1} \oplus_{1} \mathbb{R}$ is true, because $\operatorname{Var}(f)=\left\|f^{\prime}\right\|_{L_{1}}$ for $f \in \mathrm{AC} \subset \mathrm{BV}$.

Theorem 2.2 allows us to say more on the inclusion $\mathrm{BV}_{p} \subset C$ for $p>1$. In fact, for $f \in \mathrm{BV}_{p}$ and $0 \leq t \leq 1$ we have

$$
|f(t)|-|f(0)| \leq\left\|f^{\prime}\right\|_{L_{1}} \leq\left\|f^{\prime}\right\|_{L_{p}}
$$

and so $\|f\|_{C} \leq\|f\|_{\mathrm{BV}_{p}}$, by (2.3). In this way we have proved the following 
Corollary 2.3. For $p>1$ the space $\mathrm{BV}_{p}$ with the norm (2.2) is continuously embedded into the space $C$ of continuous functions with the maximum norm and embedding constant 1 .

Definition 2.1 and Theorem 2.2 were later extended by Yu.T. Mevdvedev who replaced Lebesgue spaces by Orlicz spaces. Recall that a mapping $\phi:[0, \infty) \rightarrow$ $[0, \infty)$ is said to be a Young function if $\phi$ is continuous, convex, and satisfies $\phi(0)=0, \phi(t)>0$ for $t>0$, and $\phi(t) \rightarrow \infty$ as $t \rightarrow \infty$. Given a Young function, the corresponding Orlicz space $L_{\phi}$ consists of all measurable functions $f:[0,1] \rightarrow \mathbb{R}$ for which there exists $\lambda>0$ such that

$$
\int_{0}^{1} \phi\left(\frac{|f(x)|}{\lambda}\right) d x \leq 1 .
$$

This space with the Luxemburg norm

$$
\|f\|_{L_{\phi}}=\inf \left\{\lambda>0: \int_{0}^{1} \phi\left(\frac{|f(x)|}{\lambda}\right) d x \leq 1\right\}
$$

becomes a Banach space, see e.g. [16], [23]. To avoid $L_{1}$-type spaces for which Theorem 2.2 fails, we will assume throughout that the Young function $\phi$ satisfies the so-called condition $\infty_{1}$ which means that

$$
\lim _{t \rightarrow \infty} \frac{\phi(t)}{t}=\infty
$$

Under this condition, $L_{\phi}$ is a proper subspace of $L_{1}$. A useful estimate for Young functions satisfying condition $\infty_{1}$ is Jensen's inequality

$$
\phi\left(\frac{\int_{0}^{1} \alpha(t) u(t) d t}{\int_{0}^{1} \alpha(t) d t}\right) \leq \frac{\int_{0}^{1} \alpha(t) \phi(u(t)) d t}{\int_{0}^{1} \alpha(t) d t},
$$

where $u$ and $\alpha$ are nonegative integrable functions and $\|\alpha\|_{L_{1}}>0$. We also assume that $\phi$ satisfies the $\Delta_{2}$-condition, i.e. there exist $C>0$ and $T>0$ such that $\phi(2 t) \leq C \phi(t)$ for $t \geq T$. From this it follows that, more generally, for each $\eta>0$ we can find $C=C(\eta)>0$ and $T=T(\eta)>0$ such that

$$
\phi(\eta t) \leq C(\eta) \phi(t) \quad(t \geq T(\eta)) .
$$

For a finite measure, as the Lebesgue measure on $[0,1]$, it is well-known that the $\Delta_{2}$-condition for $\phi$ implies that

$$
\int_{0}^{1} \phi\left(\frac{|f(x)|}{\lambda}\right) d x<\infty
$$

for every $\lambda>0$ if $f \in L_{\phi}$. Moreover, in this case the space $L_{\infty}$ is dense in $L_{\phi}$.

Of course, the simplest example of a Young function satisfying both conditions $\infty_{1}$ and $\Delta_{2}$ is $\phi(t)=t^{p}$ for $p>1$; however, other choices of $\phi$ are also 
important in applications. The Riesz variation (2.1) has been extended to Orlicz spaces by Medvedev [19] in the following way.

Definition 2.4. Given a Young function $\phi$, the total Riesz-Medvedev variation of a function $f:[0,1] \rightarrow \mathbb{R}$ is defined to be the (possibly infinite) number

$$
\operatorname{Var}_{\phi}(f):=\sup \left\{\sum_{j=1}^{m} \phi\left(\frac{\left|f\left(t_{j}\right)-f\left(t_{j-1}\right)\right|}{t_{j}-t_{j-1}}\right)\left(t_{j}-t_{j-1}\right): P=\left\{t_{0}, \ldots, t_{m}\right\}\right\},
$$

where the supremum is taken over all partitions $P=\left\{t_{0}, \ldots, t_{m}\right\}$ of the interval $[0,1]$. In case $\operatorname{Var}_{\phi}(f)<\infty$ we say that $f$ has bounded Riesz-Medvedev variation on $[0,1]$ and write $f \in \mathrm{BV}_{\phi}([0,1])$.

Again, it is not hard to show that $\mathrm{BV}_{\phi}$, equipped with the norm

$$
\|f\|_{\mathrm{BV}_{\phi}}:=|f(0)|+\inf \left\{\lambda>0: \operatorname{Var}_{\phi}(f / \lambda) \leq 1\right\} \quad\left(f \in \mathrm{BV}_{\phi}\right),
$$

is a Banach space. The following theorem [19] is parallel to Theorem 2.2.

THEOREM 2.5 (Medvedev). Let $\phi$ be a Young function which satisfies condition $\infty_{1}$. Then, a function $f$ belongs to $\mathrm{BV}_{\phi}$ if and only if $f$ is absolutely continuous and $f^{\prime} \in L_{\phi}$. Moreover,

$$
\operatorname{Var}_{\phi}(f)=\int_{0}^{1} \phi\left(\left|f^{\prime}(x)\right|\right) d x
$$

and so

$$
\|f\|_{\mathrm{BV}_{\phi}}=|f(0)|+\left\|f^{\prime}\right\|_{L_{\phi}} .
$$

We will use Theorem 2.5 in the next section for proving an existence result for solutions of the Hammerstein equation (1.1) in $\mathrm{BV}_{\phi}$. To this end, we need the following compactness criterion in Orlicz spaces.

THEOREM 2.6. A bounded subset $M \subset L_{\phi}$ is relatively compact if and only if

$$
\lim _{\tau \rightarrow 0} \sup _{f \in M}\left\|f-f_{\tau}\right\|_{L_{\phi}}=0,
$$

where $f_{\tau}(t)=f(t+\tau)$ for $0 \leq t+\tau \leq 1$, and $f_{\tau}(t)=0$ otherwise.

Theorem 2.6 is proved in [14, Theorem 7] and generalizes the classical Riesz compactness criterion [26] in $L_{p}$ spaces.

\section{First existence theorem}

In this section we assume throughout that $\phi$ is a Young function which satisfies both conditions $\infty_{1}$ and $\Delta_{2}$. Moreover, without loss of generality we suppose that $\phi$ is normalized, i.e. $\phi(1)=1$. Note that, for a normalized $\phi$ satisfying $\infty_{1}$, Jensen's inequality implies that $\|f\|_{L_{1}} \leq\|f\|_{L_{\phi}}$ for every $f \in L_{\phi}$. 
Under these assumptions on $\phi$, our main goal is now to study the solvability of equation (1.1) in the Riesz-Medvedev space $\mathrm{BV}_{\phi}$. To this end, we impose the following conditions.

Assumption 3.1. The function $k:[0,1] \times[0,1] \rightarrow \mathbb{R}$ is measurable with $k\left(s_{0}, \cdot\right) \in L_{1}$ for some $s_{0} \in[0,1]$, and $k(\cdot, t) \in \mathrm{BV}_{\phi}$ for almost all $t \in[0,1]$.

Assumption 3.2. The function $v:[0,1] \rightarrow \mathbb{R}$ defined by

$$
v(t):=\operatorname{Var}_{\phi}(k(\cdot, t)) \quad(0 \leq t \leq 1)
$$

belongs to $L_{1}$

Assumption 3.3. The partial derivative $k_{s}(\cdot, t)$ exists and is continuous for almost all $t \in[0,1]$, and the function $w:[0,1] \rightarrow \mathbb{R}$ defined by

$$
w(t):=\left\|k_{s}(\cdot, t)\right\|_{C} \quad(0 \leq t \leq 1)
$$

belongs to $L_{\phi}$.

Assumption 3.4. The function $h:[0,1] \times \mathbb{R} \rightarrow \mathbb{R}$ is continuous and bounded.

Observe that Assumption 3.1 implies that $\operatorname{Var}_{\phi}(k(\cdot, t))<\infty$ for almost all $t \in[0,1]$, so from Medvedev's theorem we conclude that $k(\cdot, t)$ is absolutely continuous with $k_{s}(\cdot, t) \in L_{\phi}$ for almost all $t \in[0,1]$. Of course, Assumptions 3.2 and 3.3 are stronger. In fact, under Assumption 3.2, the integrability of $k\left(s_{0}, \cdot\right) \in L_{1}$ for some $s_{0} \in[0,1]$ implies the integrability of $k(s, \cdot) \in L_{1}$ for all $s \in[0,1]$, as is shown by the following:

Lemma 3.5. Under Assumptions 3.1 and 3.2 , the function $k(s, \cdot)$ belongs to $L_{1}$ for every $s \in[0,1]$.

Proof. Since $k$ is a Lebesgue measurable function on $[0,1] \times[0,1]$ we know that the one-variable function $t \mapsto k(s, t)$ is Lebesgue measurable for almost all $s \in[0,1]$. Thus, for an arbitrary $s \in[0,1]$ there exists a sequence $\left\{s_{n}\right\}$ convergent to $s$ such that $k\left(s_{n}, \cdot\right)$ is measurable. Since $k(\cdot, t)$ is continuous for almost all $t \in[0,1]$ we easily obtain that $k(s, \cdot)$ is Lebesgue measurable.

Considering the partition $P=\left\{0, s_{0}, 1\right\}$, for every $t \in[0,1]$, we have

$$
\phi\left(\frac{\left|k\left(s_{0}, t\right)-k(0, t)\right|}{s_{0}}\right) s_{0}+\phi\left(\frac{\left|k(1, t)-k\left(s_{0}, t\right)\right|}{1-s_{0}}\right)\left(1-s_{0}\right) \leq v(t),
$$

which implies that both $\left|k\left(s_{0}, \cdot\right)-k(0, \cdot)\right|$ and $\left|k(1, \cdot)-k\left(s_{0}, \cdot\right)\right|$ belong to $L_{1}$. Thus, $k(0, \cdot)$ and $k(1, \cdot)$ are integrable. Given $s \in[0,1]$ arbitrary, a similar argument shows that $|k(s, \cdot)-k(0, \cdot)|$ belongs to $L_{1}$, and so $k(s, \cdot)$ is integrable. $\square$

Note that we cannot remove the condition $k\left(s_{0}, \cdot\right) \in L_{1}$ for at least one $s_{0} \in[0,1]$ from Assumption 3.1. Indeed, consider, for instance, the function 
$k:[0,1] \times[0,1] \rightarrow \mathbb{R}$ defined by

$$
k(s, t):= \begin{cases}s+1 / t & \text { for } 0<t \leq 1 \\ 0 & \text { for } t=0\end{cases}
$$

Then $k(s, \cdot) \notin L_{1}$ for $0 \leq s \leq 1$, but $k$ satisfies all remaining conditions from Assumptions 3.1-3.3 with $v(t)=w(t) \equiv 1$. However, it is clear that the function $t \mapsto k(s, t) h(t, f(t))$ is not, in general, integrable, even if $h$ and $f$ are very regular.

In what follows, we use the shortcut

$$
\eta:=\sup \{|h(t, u)|: 0 \leq t \leq 1, u \in \mathbb{R}\} .
$$

Moreover, by $B_{r}(X)$ we denote the closed ball of radius $r>0$ centered at zero in a normed space $X$. Under the hypotheses stated above, we may now prove the announced existence theorem.

Theorem 3.6. Let $b \in \mathrm{BV}_{\phi}$, and let Assumptions 3.1-3.4 be satisfied. Then there exists $R>0$ such that the operator (1.2) is compact and continuous and maps the ball $B_{R}\left(\mathrm{BV}_{\phi}\right)$ into itself. Consequently, equation (1.1) has a solution in this ball.

Proof. Fix $f \in \mathrm{BV}_{\phi}$. Since $k$ is measurable, $k(s, \cdot)$ belongs to $L_{1}$ for all $s \in[0,1]$, by Lemma 3.5 , and the function $H f$ is continuous and bounded, the function $t \mapsto k(s, t) h(t, f(t))$ is integrable for every $s \in[0,1]$, and so the function $g:=K H f$ in (1.2) is well-defined. We will prove that this function belongs to $\mathrm{BV}_{\phi}$ for every continuous function $f$. Indeed, for any partition $P=\left\{s_{0}, \ldots, s_{m}\right\}$ of the interval $[0,1]$ we have

$$
\frac{\left|g\left(s_{j}\right)-g\left(s_{j-1}\right)\right|}{s_{j}-s_{j-1}} \leq \eta \int_{0}^{1} \frac{\left|k\left(s_{j}, t\right)-k\left(s_{j-1}, t\right)\right|}{s_{j}-s_{j-1}} d t,
$$

with $\eta$ given by (3.3). We distinguish two cases. If

$$
\int_{0}^{1} \frac{\left|k\left(s_{j}, t\right)-k\left(s_{j-1}, t\right)\right|}{s_{j}-s_{j-1}} d t \geq T(\eta)
$$

then combining (2.6) with the Jensen inequality $(2.5)$ for $\alpha(t) \equiv 1$ we obtain

$$
\begin{aligned}
\phi\left(\frac{\left|g\left(s_{j}\right)-g\left(s_{j-1}\right)\right|}{s_{j}-s_{j-1}}\right) & \leq C(\eta) \phi\left(\int_{0}^{1} \frac{\left|k\left(s_{j}, t\right)-k\left(s_{j-1}, t\right)\right|}{s_{j}-s_{j-1}} d t\right) \\
& \leq C(\eta) \int_{0}^{1} \phi\left(\frac{\left|k\left(s_{j}, t\right)-k\left(s_{j-1}, t\right)\right|}{s_{j}-s_{j-1}}\right) d t .
\end{aligned}
$$

On the other hand, in case

$$
\int_{0}^{1} \frac{\left|k\left(s_{j}, t\right)-k\left(s_{j-1}, t\right)\right|}{s_{j}-s_{j-1}} d t<T(\eta)
$$


we immediately obtain

$$
\phi\left(\frac{\left|g\left(s_{j}\right)-g\left(s_{j-1}\right)\right|}{s_{j}-s_{j-1}}\right) \leq \phi(\eta T(\eta)),
$$

since $\phi$ is monotone. Multiplying both estimates by $s_{j}-s_{j-1}$ and summing up over $j=1, \ldots, m$, we get

$$
\begin{aligned}
\sum_{j=1}^{m} \phi & \left(\frac{\left|g\left(s_{j}\right)-g\left(s_{j-1}\right)\right|}{\left.s_{j}-s_{j-1}\right)}\right)\left(s_{j}-s_{j-1}\right) \\
\leq & C(\eta) \sum_{j=1}^{m} \int_{0}^{1} \phi\left(\frac{\left|k\left(s_{j}, t\right)-k\left(s_{j-1}, t\right)\right|}{s_{j}-s_{j-1}}\right)\left(s_{j}-s_{j-1}\right) d t \\
& +\sum_{j=1}^{m} \phi(\eta T(\eta))\left(s_{j}-s_{j-1}\right) \\
\leq & C(\eta) \int_{0}^{1} \operatorname{Var}_{\phi}(k(\cdot, t)) d t+\phi(\eta T(\eta))=C(\eta)\|v\|_{L_{1}}+\phi(\eta T(\eta)),
\end{aligned}
$$

with $v$ given by (3.1), and so

$$
\operatorname{Var}_{\phi}(g) \leq C(\eta)\|v\|_{L_{1}}+\phi(\eta T(\eta)) .
$$

Furthermore,

$$
|g(0)|=\left|\int_{0}^{1} k(0, t) h(t, f(t)) d t\right| \leq \eta\|k(0, \cdot)\|_{L_{1}}<\infty,
$$

by Lemma 3.5. Thus, if we choose

$$
R \geq C(\eta)\|v\|_{L_{1}}+\phi(\eta T(\eta))+\eta\|k(0, \cdot)\|_{L_{1}}+\|b\|_{\mathrm{BV}_{\phi}},
$$

we see that the operator $A$ from (1.2) maps the ball $B_{R}\left(\mathrm{BV}_{\phi}\right)$ into itself.

To prove that this operator is compact we use Theorem 2.5 and the compactness criterion given in Theorem 2.6. So we have to show that

$$
\lim _{\tau \rightarrow 0} \sup \left\{\left\|(H K f)^{\prime}-(H K f)_{\tau}^{\prime}\right\|_{L_{\phi}}:\|f\|_{\mathrm{BV}_{\phi}} \leq R\right\}=0
$$

which amounts to considering the function

$$
\psi_{\tau, \varepsilon}(s, t):=\phi\left(\frac{\left|k_{s}(s, t)-k_{s}(s+\tau, t)\right|}{\varepsilon}\right)
$$

for $(s, t)$ varying over the unit square $[0,1] \times[0,1]$, where, as in Theorem 2.6, we assume that $k(s+\tau, t)=0$ if $s+\tau \notin[0,1]$. Observe that this function is measurable, because the derivative $k_{s}$ is the limit of the measurable functions

$$
k_{n}(s, t):=n k\left(s+\frac{1}{n}, t\right)-n k(s, t) \quad(n=1,2, \ldots),
$$


and $\phi$ is continuous, by assumption. Now, by applying the classical parametric derivation theorem

$$
\frac{d}{d s} \int_{0}^{1} \gamma(s, t) d t=\int_{0}^{1} \frac{\partial}{\partial s} \gamma(s, t) d t
$$

to $\gamma(s, t):=k(s, t) h(t, f(t))$, Jensen's inequality (2.5), Fubini's theorem, and the monotonicity of $\phi$, we obtain, for $\eta$ as in (3.3),

$$
\begin{aligned}
\inf \{\varepsilon>0 & \left.: \int_{0}^{1} \phi\left(\left|\frac{1}{\varepsilon} \int_{0}^{1}\left[k_{s}(s, t)-k_{s}(s+\tau, t)\right] h(t, f(t)) d t\right|\right) d s \leq 1\right\} \\
& \leq \inf \left\{\varepsilon>0: \int_{0}^{1} \phi\left(\int_{0}^{1} \eta \frac{\left|k_{s}(s, t)-k_{s}(s+\tau, t)\right|}{\varepsilon} d t\right) d s \leq 1\right\} \\
& \leq \inf \left\{\varepsilon>0: \int_{0}^{1}\left(\int_{0}^{1} \phi\left(\eta \frac{\left|k_{s}(s, t)-k_{s}(s+\tau, t)\right|}{\varepsilon}\right) d t\right) d s \leq 1\right\} \\
& \leq \eta \inf \left\{\varepsilon>0: \int_{0}^{1} \int_{0}^{1} \psi_{\tau, \varepsilon}(s, t) d s d t \leq 1\right\} .
\end{aligned}
$$

Moreover, from $\psi_{\tau, \varepsilon}(s, t) \leq \phi(2 w(t) / \varepsilon)(0 \leq s, t \leq 1)$ and Assumption 3.3 we deduce that

$$
\int_{0}^{1} \int_{0}^{1} \psi_{\tau, \varepsilon}(s, t) d s d t \rightarrow 0 \quad(\tau \rightarrow 0),
$$

by the Lebesgue Dominated Convergence theorem. Thus, for any $\varepsilon>0$ there exists $\tau_{0}>0$, depending only on $k$ and $\phi$, such that

$$
\int_{0}^{1} \int_{0}^{1} \psi_{\tau, \varepsilon}(s, t) d s d t \leq 1
$$

for $\tau \leq \tau_{0}$, which implies that $\left\|(H K f)^{\prime}-(H K f)_{\tau}^{\prime}\right\|_{L_{\phi}} \leq \varepsilon$ if $\tau \leq \tau_{0}$. Since $\tau_{0}$ is independent of $f \in B_{R}\left(\mathrm{BV}_{\phi}\right)$, we conclude that (3.4) is true.

It remains to prove that the operator $K H$ is continuous on $B_{R}\left(\mathrm{BV}_{\phi}\right)$. Fix $f_{0} \in \mathrm{BV}_{\phi}$, and let $m:=\left\|f_{0}\right\|_{C}$. The uniform continuity of $h$ on the compact rectangle $[0,1] \times[-m-1, m+1]$ implies that for a given $\varepsilon>0$ we can find $\delta \in(0,1)$ such that

$$
|h(s, u)-h(t, v)| \leq \varepsilon^{\prime}:=\min \left\{\frac{\varepsilon}{2\|w\|_{L_{\phi}}}, \frac{\varepsilon}{2\|k(0, \cdot)\|_{L_{1}}}\right\}
$$

for $|s-t|<\delta,|u|,|v| \leq m+1$, and $|u-v| \leq \delta$, where $w$ is taken from (3.2). Now take any $f \in \mathrm{BV}_{\phi}$ with $\left\|f-f_{0}\right\|_{\mathrm{BV}_{\phi}} \leq \delta$; we claim that $\left\|g-g_{0}\right\|_{\mathrm{BV}_{\phi}} \leq \varepsilon$, where $g_{0}:=K H f_{0}$ and $g:=K H f$. In fact, for every $t \in[0,1]$ we have

$$
\left|f(t)-f_{0}(t)\right| \leq\left|f(0)-f_{0}(0)\right|+\left\|f^{\prime}-f_{0}^{\prime}\right\|_{L_{1}} \leq\left|f(0)-f_{0}(0)\right|+\left\|f^{\prime}-f_{0}^{\prime}\right\|_{L_{\phi}} \leq \delta
$$


by (2.8). Thus, $|f(t)| \leq m+1$ and so $\left|h(t, f(t))-h\left(t, f_{0}(t)\right)\right| \leq \varepsilon^{\prime}$ for every $t \in[0,1]$. Together with Jensen's inequality and the parametric derivation theorem (3.6), applied to $\gamma(s, t):=k(s, t)\left[h(t, f(t))-h\left(t, f_{0}(t)\right)\right]$, this implies that

$$
\begin{aligned}
\int_{0}^{1} \phi\left(\frac{\left|g^{\prime}(s)-g_{0}^{\prime}(s)\right|}{\varepsilon / 2}\right) d s \leq \int_{0}^{1} \phi\left(\frac{\left\|k_{s}(s, \cdot)\right\|_{L_{1}}}{\|w\|_{L_{\phi}}}\right) d s \\
\leq \int_{0}^{1} \phi\left(\int_{0}^{1} \frac{w(t)}{\|w\|_{L_{\phi}}} d t\right) d s \leq \int_{0}^{1} \int_{0}^{1} \phi\left(\frac{w(t)}{\|w\|_{L_{\phi}}}\right) d t d s \leq 1,
\end{aligned}
$$

hence $\left\|g^{\prime}-g_{0}^{\prime}\right\|_{L_{\phi}} \leq \varepsilon / 2$. Analogously,

$$
\left|g(0)-g_{0}(0)\right| \leq \int_{0}^{1} \frac{\varepsilon|k(0, t)|}{2\|k(0, \cdot)\|_{L_{1}}} d t=\frac{\varepsilon}{2} .
$$

Combining these two estimates we see that $\left\|g-g_{0}\right\|_{\mathrm{BV}_{\phi}} \leq \varepsilon$, and so we are done.

\section{Second existence theorem}

Of course, the global boundedness condition on $h:[0,1] \times \mathbb{R} \rightarrow \mathbb{R}$ is a rather strong requirement. In the following Theorem 4.2, which we state for the space $\mathrm{BV}_{p}$ we will prove the existence of solutions of equation (1.1) under a weaker assumption. We suppose, as before, that the above Assumptions 3.1-3.3 are fulfilled for $\phi(t)=t^{p}$, but instead of 3.4 we assume

Assumption 4.1. The function $h:[0,1] \times \mathbb{R} \rightarrow \mathbb{R}$ is continuous and satisfies:

$$
|h(t, u)|^{p} \leq A|u|+B \quad(0 \leq t \leq 1, u \in \mathbb{R})
$$

for some constants $A$ and $B$ in $(0, \infty)$.

Then we have the following:

Theorem 4.2. Let $b \in \mathrm{BV}_{p}$, and let Assumptions 3.1-3.3 and 4.1 be satisfied. Then there exists $R>0$ such that the operator (1.2) is continuous and compact and maps the ball $B_{R}\left(\mathrm{BV}_{p}\right)$ into itself. Consequently, equation (1.1) has a solution in this ball.

Proof. The proof is similar to that of Theorem 3.6, so we only sketch the idea. First of all, note that the continuity of $h$ implies that $h$ is bounded on bounded sets which implies that the function $t \mapsto k(s, t) h(t, f(t))$ is integrable for any continuous function $f$. Fix $f \in B_{r}\left(\mathrm{BV}_{p}\right)$ and put again $K H f=: g$.

By Assumption 4.1, we have

$$
|h(t, f(t))|^{p} \leq A r+B \quad\left(0 \leq t \leq 1, f \in B_{r}\left(\mathrm{BV}_{p}\right) \subset B_{r}(C)\right),
$$


where we have used Corollary 2.3 in the inclusion $B_{r}\left(\mathrm{BV}_{p}\right) \subset B_{r}(C)$. Following the same argument as in the proof of Theorem 3.6, we deduce that

$$
\operatorname{Var}_{p}(g) \leq(A r+B) \int_{0}^{1} \operatorname{Var}_{p}(k(\cdot, t)) d t=(A r+B)\|v\|_{L_{1}} \quad\left(f \in B_{r}\left(\mathrm{BV}_{p}\right)\right) .
$$

Furthermore, since $k(0, \cdot) \in L_{1}$ we have

$$
|g(0)|=\left|\int_{0}^{1} k(0, t) h(t, f(t)) d t\right| \leq(A r+B)^{1 / p}\|k(0, \cdot)\|_{L_{1}} .
$$

Thus, choosing for a given $b \in \mathrm{BV}_{p}$, a number $R$ large enough such that

$$
R>(A R+B)^{1 / p}\|v\|_{L_{1}}^{1 / p}+(A R+B)^{1 / p}\|k(0, \cdot)\|_{L_{1}}+\|b\|_{\mathrm{BV}_{p}},
$$

it follows that the operator (1.2) maps the ball $B_{R}\left(\mathrm{BV}_{p}\right)$ into itself. The compactness and continuity of the operator (1.2) on this ball is proved exactly as in Theorem 3.6.

\section{Concluding remarks}

We make some comments on Theorems 3.6 and 4.2. First of all, observe that our hypotheses 3.1-3.4 do not imply that the operators (1.3) and (1.4) map the space $\mathrm{BV}_{\phi}$ (in particular, $\mathrm{BV}_{p}$ ) into itself, in contrast to what is often assumed for proving existence. Consider, for example, the special choice

$$
\phi(t):=t^{p}, \quad k(s, t):=s^{\alpha} t^{\beta}, \quad h(u):=|u|^{\gamma} .
$$

Then Assumptions 3.1 and 3.2 are satisfied for $(\alpha-1) p>-1$ and $\beta>-1$. Assumption 3.3 is satisfied for $\alpha \geq 1$ and $\beta p>-1$ (which implies Assumptions 3.1 and 3.2), and Assumption 4.1 for $0 \leq \gamma p<1$. However, Assumption 3.4 is satisfied only for $\gamma=0$, so in case $0<\gamma p<1$ Theorem 4.2 applies, but Theorem 3.6 does not.

As was shown in [21] (see also [3, Theorem 5.10]), the composition operator $H$ maps the space $\mathrm{BV}_{p}$ into itself if and only if $h \in \operatorname{Lip}_{\text {loc }}(\mathbb{R})$ which here means $\gamma \geq 1$. But this is excluded by the condition $\gamma p \leq 1$, since $p>1$. Consequently, in this example (assuming $0<\gamma p<1, \beta p>-1$, and $\alpha \geq 1$ ) the operator (1.3) never maps $\mathrm{BV}_{p}$ into itself, but the operator (1.2) does.

Now comes an important point which is concerned with the use of fixed point theorems. To prove existence of solutions of (1.1), Banach's contraction mapping theorem is frequently used, because it also gives uniqueness and constructibility. This works well in the spaces $C, L_{p}$, or $L_{\phi}$. However, a contraction hypothesis on the operator $H$ in spaces like $\mathrm{BV}_{p}$ or $\mathrm{BV}_{\phi}$ leads to a strong degeneracy of the corresponding function $h$; for the space $\mathrm{BV}_{p}$ this was shown in [22], for the space $\mathrm{BV}_{\phi}$ in [20]. This is the reason why we applied Schauder's fixed point principle in Theorems 3.6 and 4.2, rather than Banach's contraction mapping theorem. 
However, we can do better. Both Banach's and Schauder's theorems are in a certain sense contained in a more general fixed point principle proved by G. Darbo [13] in 1955. Recall that the Kuratowski measure of noncompactness $\gamma$ associates to each bounded subset $M$ of a Banach space $X$ the nonnegative number [17]

(5.1) $\gamma(M):=\inf \{d>0: M$ may be covered by finitely many sets

$$
\left.M_{1}, \ldots, M_{k} \text { satisfying } \operatorname{diam}\left(M_{j}\right) \leq d \text { for } j=1, \ldots, k\right\} .
$$

This name is of course motivated by the fact that $\gamma(M)=0$ if and only if $M$ is relatively compact. A continuous operator $A: B_{R}(X) \rightarrow X$ is called condensing if there exists $q<1$ such that $\gamma(A(M)) \leq q \gamma(M)$ for every $M \subseteq B_{R}(X)$. Darbo's fixed point principle states that a condensing operator which maps a ball $B_{R}(X)$ into itself has a fixed point. Since both contractions and compact operators are obviously condensing, Darbo's theorem bridges the gap between the apparently quite different fixed point principles by Banach and Schauder. A thorough presentation of the theory and applications of this fixed point theorem may be found, for example, in [1], [2], [4], [5].

In our context Darbo's theorem may be applied as follows. It is well-known that the function

$$
\gamma^{*}(M):=\lim _{\tau \rightarrow 0} \sup _{f \in M}\left\|f-f_{\tau}\right\|_{L_{p}} \quad\left(M \subset L_{p} \text { bounded }\right)
$$

is equivalent to the Kuratowski measure of noncompactness (5.1) in the Lebesgue space $L_{p}$ in the sense that $\gamma(M) \leq 2 \gamma^{*}(M) \leq 4 \gamma(M)$ for each bounded subset $M$. In particular, this explains why Theorem 2.6 characterizes precisely the relatively compact subsets $M \subset L_{p}$. Now, relation (2.3) suggests to consider, for $p>1$, the function

$$
\gamma^{*}(M):=\lim _{\tau \rightarrow 0} \sup _{f \in M}\left\|f^{\prime}-f_{\tau}^{\prime}\right\|_{L_{p}} \quad\left(M \subset \mathrm{BV}_{p} \text { bounded }\right)
$$

which is in turn equivalent to the Kuratowski measure of noncompactness in $\mathrm{BV}_{p}$, and to find conditions on $h$ and $k$ under which the operator (1.2) is condensing in the space $\mathrm{BV}_{p}$ for $p>1$. This makes it possible to impose milder conditions than our Assumptions 3.1-3.4 and 4.1 above to prove existence of solutions.

Acknowledgements. The authors are indebted to the referees for some useful remarks which essentially improved the paper.

\section{REFERENCES}

[1] R.R. Akhmerov, M.I. Kamenskil̆, A.S. Potapov, A.E. Rodkina and B.N. SadovskiĬ, Measures of Noncompactness and Condensing Operators (Russian), Nauka, Novosibirsk 1986; Engl. transl.: Birkhäuser, Basel 1992. 
[2] J. ApPelL, Measures of noncompactness, condensing operators and fixed points: An application-oriented survey, Fixed Point Theory (Cluj) 6 (2005), 157-229.

[3] J. Appell, J. Banaś and N. Merentes, Bounded Variation and Around, De Gruyter Ser. Nonlinear Anal. Appl. 17, De Gruyter, Berlin 2014

[4] J.M. Ayerbe Toledano, T. Domínguez Benavides And G. López Acedo, Measures of Noncompactness in Metric Fixed Point Theory, Birkhäuser, Basel 1997.

[5] J. Banaś And K. Goebel, Measures of Noncompactness in Banach Spaces, Lect. Notes Pure Appl. Math. 60, M. Dekker, New York 1980.

[6] D. Bugajewska, On the superposition operator in the space of functions of bounded variation, revisited, Math. Comp. Modelling 52 (2010), 791-796.

[7] D. Bugajewska And D. Bugajewski, On nonlinear integral equations and nonabsolutely convergent integrals, J. Dynam. Systems Appl. 14 (2005), 135-148.

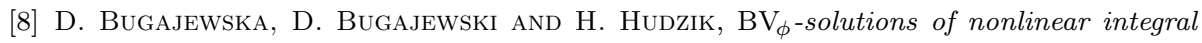
equations, J. Math. Anal. Appl. 287 (2003), 265-278.

[9] D. Bugajewska, D. Bugajewski and G. Lewicki, On nonlinear integral equations in spaces of functions of bounded generalized $\varphi$-variation, J. Integral Equtions Appl. 21 (1) (2009), 1-20.

[10] D. Bugajewska And D. O'REgan, On nonlinear integral equations and $\Lambda$-bounded variation, Acta Math. Hung. 107 (4) (2005), 295-306.

[11] D. Bugajewski, On BV-solutions of some nonlinear integral equations, Integral Equations Operator Theory 46 (2003), 387-398.

[12] D. Bugajewski and D. O'Regan, Existence results for BV-solutions of nonlinear integral equations, J. Integral Equations Appl. 15 (4) (2003), 343-357.

[13] G. Darbo, Punti uniti in trasformazioni a codominio non compatto. Rend. Sem. Mat. Univ. Padova 24 (1955), 84-92.

[14] Yu.I. Gribanov and P.K. Belobrov, On a class of Banach spaces of functions, Izv. Vysh. Uchebn. Zaved. Mat. 35 (4) (1963), 44-55 (Russian).

[15] C. Jordan, Sur la série de Fourier, C.R. Acad. Sci. Paris 2 (1881), 228-230.

[16] M.A. Krasnosel'skǐ and Ya.B. RutitskiĬ, Convex Functions and Orlicz Spaces (Russian), Fizmatgiz., Moscow 1958; Engl. transl.: Noordhoff, Groningen 1961.

[17] K. Kuratowski, Sur les espaces complètes, Fund. Math. 15 (1934), 301-335.

[18] J.J. Levin, On a nonlinear Volterra equations, J. Math. Anal. Appl. 39 (1972), 458-476.

[19] YU. T. Mevdvedev, A generalization of a certain theorem of Riesz (Russian), Uspekhi Mat. Nauk. 6 (1953), 115-118.

[20] N. Merentes, On a characterization of Lipschitzian operators of substitution in the space of bounded Riesz $\varphi$-variation, Ann. Univ. Sci. Budapest 34 (1991), 139-144.

[21] _ On the composition operator in $R V_{\varphi}[a, b]$, Collect. Math. 46 (3) (1995), 231-238.

[22] N. Merentes AND S. Rivas, On characterization of the Lipschitzian composition operator between spaces of functions of bounded p-variation, Czechosl. Math. J. 45, (4) (1995), $627-637$.

[23] M.M. RaO And Z.D. Ren, Theory of Orlicz spaces, M. Dekker, New York 1991.

[24] F. RIESZ, Untersuchungen über Systeme integrierbarer Funktionen, Math. Annalen 69 (1910), 449-497.

[25] _ Sur certain systèmes singulieres d'équations intégrales, Ann. Sci. Ecole Norm. Sup. Paris 28 (1911), 33-68.

[26] _ Sur les ensembles compacts de fonctions sommables, Acta Sci. Math. (Szeged) 6 (2-3) (1932-34), 136-142. 
[27] D. Waterman, On the summability of Fourier series of functions of $\Lambda$-bounded variation, Studia Math. 55 (1976), 87-95.

[28] _ On $\Lambda$-bounded variation, Studia Math. 57 (1976), 33-45.

[29] (1) (1979), 119-123.

[30] _ Estimating functions by partial sums of their Fourier series, J. Math. Anal. Appl. 87 (1982), 51-57.

[31] N. Wiener, The quadratic variation of a function and its Fourier coefficients, J. Math. Phys. MIT 3 (1924), 73-94.

Manuscript received September 3, 2014 accepted August 26, 2015

JÜRGEN APPELL

Institut für Mathematik

Universität Würzburg

D-97074 Würzburg, GERMANY

E-mail address: jurgen@dmuw.de

Tomás Domínguez Benavides

Facultad de Matemáticas

Universidad de Sevilla

41013-Sevilla, SPAIN

E-mail address: tomasd@us.es 